\title{
Supersymmetric Form of the String Action in the Presence of the Gauge Field
}

\author{
Davoud Kamani \\ Faculty of Physics, Amirkabir University of Technology (Tehran Polytechnic) \\ P.O.Box: 15875-4413, Tehran, Iran \\ e-mail: kamani@aut.ac.ir
}

\begin{abstract}
In this manuscript we study the superstring theory with an Abelian worldsheet gauge field. The components of the gauge field appear as a space and a time coordinates. We call them "fictitious coordinates". The worldsheet supersymmetry and the Poincaré symmetry of this model will be analyzed. The $T$-duality and quantization of the two fictitious coordinates are also obtained.
\end{abstract}

PACS number: 11.25.-w; 11.30.Cp

Keywords: Superstring; Gauge field; Symmetry. 


\section{Introduction}

Gauged actions in the string theory have been studied from various points of view [1]. We introduce an Abelian worldsheet gauge field in the superstring action. Our motivation is as follows. The S-duality of the type IIB theory implies that fundamental string of IIB is S-dual to the D-string. Thus, according to the observation in ref. [2], the worldsheet fields comprise not only the target space coordinates, but also an $S L(2 ; R)$ doublet of Abelian gauge fields. In addition, the worldsheet with gauge field in the Matrix string theory also has been studied [3].

We are interested in the case of the $U(1)$ gauge field, which exists on the string worldsheet, to be independent degree of freedom. Therefore, the square form of the corresponding field strength appears in the string action. The gauged action of the superstring enables us to build two worldsheet fields from the components of the gauge field. They appear as the space and time coordinates. Since they are not actual coordinates, we call them "fictitious coordinates". The fictitious space coordinate is hidden. In other words, from the quantization point of view, we observe that it has to be compact. We study the various symmetries, T-duality, and quantization of this extended action of the superstring.

This paper is organized as follows. In Sec. 2, the superstring action in the presence of the worldsheet gauge field $A_{a}(\sigma, \tau)$ will be obtained. In Sec. 3, the components of the field $A^{a}$, as the fictitious coordinates, and also their super-partners will be studied. In Sec. 4, some symmetries of the model will be discussed. In Sec. 5, the solutions of the fictitious coordinates and some electric fields will be obtained. In Sec. 6, the effects of the T-duality on the fictitious coordinates and also their quantizations will be analyzed.

\section{The gauged action of the superstring}

The action of the superstring with the worldsheet supersymmetry is

$$
S_{0}=-\frac{1}{4 \pi \alpha^{\prime}} \int d^{2} \sigma\left(\partial^{a} X^{\mu} \partial_{a} X_{\mu}-i \bar{\psi}^{\mu} \rho^{a} \partial_{a} \psi_{\mu}\right),
$$

where we have the following notations

$$
\rho^{0}=\left(\begin{array}{cc}
0 & -i \\
i & 0
\end{array}\right) \quad, \quad \rho^{1}=\left(\begin{array}{cc}
0 & i \\
i & 0
\end{array}\right)
$$

for the matrices $\left\{\rho^{a}\right\}$ in the Majorana basis, and $\eta_{a b}=\operatorname{diag}(-1,1)$ and $\eta_{\mu \nu}=\operatorname{diag}(-1,1, \ldots, 1)$ for the worldsheet and spacetime metrics. The supersymmetry transformations for this ac- 
tion are

$$
\begin{aligned}
& \delta X^{\mu}=\bar{\eta} \psi^{\mu}, \\
& \delta \psi^{\mu}=-i \rho^{a} \partial_{a} X^{\mu} \eta,
\end{aligned}
$$

where $\eta$ is a constant infinitesimal Majorana spinor. The supercurrent, associated with these transformations, is

$$
j_{a}=\frac{1}{2} \rho^{b} \rho_{a} \psi^{\mu} \partial_{b} X_{\mu}
$$

which is a conserved current, i.e. $\partial^{a} j_{a}=0$.

To obtain a supersymmetric action with the gauge field, we use the superfields in the worldsheet superspace. In other words, the bosonic action

$$
S_{1}=-\int d^{2} \sigma\left(\frac{1}{4 \pi \alpha^{\prime}} \partial_{a} X^{\mu} \partial^{a} X_{\mu}+\frac{1}{4 g^{2}} F_{a b} F^{a b}\right)
$$

should be supersymmetrized. The factor $g$ is gauge coupling constant and the field strength is $F_{a b}=\partial_{a} A_{b}-\partial_{b} A_{a}$. This action has the gauge symmetry. Therefore, we have the condition

$$
\partial_{a} A^{a}=0
$$

Note that the worldsheet field $A_{a}$ is not pull-back of a spacetime gauge field $A_{\mu}$.

The bosonic fields $X^{\mu}$ and $A^{a}$ should be replaced by the superfields

$$
\begin{aligned}
& Y^{\mu}\left(\sigma, \tau ; \theta^{1}, \theta^{2}\right)=X^{\mu}(\sigma, \tau)+\bar{\theta} \psi^{\mu}(\sigma, \tau)+\frac{1}{2} \bar{\theta} \theta B^{\mu}(\sigma, \tau), \\
& \mathcal{A}^{a}\left(\sigma, \tau ; \theta^{1}, \theta^{2}\right)=A^{a}(\sigma, \tau)+\bar{\theta} \rho^{a} \chi(\sigma, \tau)+\frac{1}{2} \bar{\theta} \theta W^{a}(\sigma, \tau),
\end{aligned}
$$

where $B^{\mu}$ and $W^{a}$ are auxiliary fields and the Majorana spinor $\chi$ is the super-partner of $A_{a}$. The Grassmannian coordinates $\theta^{1}$ and $\theta^{2}$ form a Majorana spinor $\theta=\left(\begin{array}{c}\theta^{1} \\ \theta^{2}\end{array}\right)$. Thus, the term such as $\bar{\theta} \rho^{a} \theta u(\sigma, \tau)$ is zero and hence does not appear in the superfield (8).

The derivative $\partial_{a}$ also should be changed. We introduce the following superspace covariant derivative

$$
\begin{aligned}
& \mathcal{D}^{a}=k \varepsilon^{a b} \rho_{b} D, \\
& D=\frac{\partial}{\partial \bar{\theta}}-i \rho^{a} \theta \partial_{a},
\end{aligned}
$$


where $\varepsilon^{01}=-\varepsilon^{10}=1$ and $k$ is a constant which will be specified. For a superfield $Z$ its derivative $D Z$ also is superfield. On the other hand, the transformation $\delta Z=\bar{\epsilon} Q(Z)$ implies $\delta(D Z)=\bar{\epsilon} Q(D Z)$. The operator

$$
Q=\frac{\partial}{\partial \bar{\theta}}+i \rho^{a} \theta \partial_{a}
$$

is generator of the supersymmetry on the superspace. Similarly, $\mathcal{D}^{a} Z$ also is a superfield. That is, it transforms in the same way

$$
\delta\left(\mathcal{D}^{a} Z\right)=\bar{\epsilon} Q\left(\mathcal{D}^{a} Z\right)
$$

Therefore, the derivative $\mathcal{D}^{a}$ enables us to write the supersymmetric form of the action (5). For finding the constant $k$ we demand the property

$$
\overline{\mathcal{D}}^{a} Y_{\mu} \mathcal{D}_{a} Y^{\mu}=\bar{D} Y_{\mu} D Y^{\mu}
$$

This gives $k \in\left\{ \pm \frac{1}{\sqrt{2}}, \pm \frac{i}{\sqrt{2}}\right\}$. Note that the definition $\mathcal{D}^{a}=k \rho^{a} D$ does not satisfy the equation (12).

Adding all these together we obtain the following supersymmetric action

$$
S=\int d^{2} \sigma d^{2} \theta\left(\frac{i}{8 \pi \alpha^{\prime}} \overline{\mathcal{D}}^{a} Y_{\mu} \mathcal{D}_{a} Y^{\mu}+\frac{i}{4 g^{2}} \overline{\mathcal{F}}_{a b} \mathcal{F}^{a b}\right)
$$

The superfield strength $\mathcal{F}_{a b}$ has the definition

$$
\mathcal{F}_{a b}=\mathcal{D}_{a} \mathcal{A}_{b}-\mathcal{D}_{b} \mathcal{A}_{a}
$$

After integration over the Grassmannian coordinates $\theta^{1}$ and $\theta^{2}$, this action takes the form

$$
S=\int d^{2} \sigma\left(-\frac{1}{4 \pi \alpha^{\prime}}\left(\partial_{a} X^{\mu} \partial^{a} X_{\mu}-i \bar{\psi}^{\mu} \rho^{a} \partial_{a} \psi_{\mu}-B^{\mu} B_{\mu}\right)-\frac{1}{2 g^{2}}\left(\partial_{a} A^{b} \partial^{a} A_{b}-W_{a} W^{a}\right)\right) .
$$

As we see, the gagino field $\chi$ from the two-dimensional action disappeared. In addition, according to the condition (6), the term $\partial_{a} A^{b} \partial_{b} A^{a}$ is double total derivative $\partial_{a} \partial_{b}\left(A^{a} A^{b}\right)$ and hence it also has been ignored.

\section{A new form for the gauged action}

In the action (15) the kinetic terms of the fields $X^{\mu}$ and $A^{b}$ have the same feature. In other words, $A^{0}$ and $A^{1}$ have the roles of the time and space coordinates. Let $\left\{X^{a}\right\}$ denote the coordinates of this fictitious $1+1$ dimensional spacetime. Thus, we have the field redefinition

$$
X^{a}=\frac{\sqrt{2 \pi \alpha^{\prime}}}{g} A^{a} .
$$


In addition, the auxiliary field $B^{a}$ also is scaled in the same way

$$
B^{a}=\frac{\sqrt{2 \pi \alpha^{\prime}}}{g} W^{a}
$$

According to these definitions, the action (15) can be written as

$$
S=-\frac{1}{4 \pi \alpha^{\prime}} \int d^{2} \sigma\left(\partial_{a} X^{M} \partial^{a} X_{M}-i \bar{\psi}^{\mu} \rho^{a} \partial_{a} \psi_{\mu}-B^{M} B_{M}\right)
$$

where $M \in\{\mu, a\}$, and we shall use the convention $a \in\{0,1\}$ and $\mu \in\left\{0^{\prime}, 1^{\prime}, \ldots, 9^{\prime}\right\}$. Since both $X^{a}$ and $\sigma^{a}$ carry the worldsheet index, the partial derivative $\partial_{a}$ always shows derivative with respect to $\sigma^{a}$. The bosonic part of this action apparently describe a 12-dimensional spacetime with the signature $10+2$ and the coordinates

$$
\left\{X^{M}\right\}=\left\{X^{\mu}\right\} \bigcup\left\{X^{a}\right\}
$$

Because the gauge condition survived, these two additional coordinates are constrained on the worldsheet. Thus, they do not describe additional $1+1$ dimensions. However, in the superstring theory the dimension of the spacetime always is $9+1$. Therefore, we call the extra dimensions as "fictitious coordinates".

The fermionic term of the action (18) also can be written with the 12-dimensional indices. For this, the Majorana spinor $\psi^{a}$ is defined by

$$
\psi^{0}=\frac{\sqrt{2 \pi \alpha^{\prime}}}{g}\left(\begin{array}{l}
\chi_{2} \\
\chi_{1}
\end{array}\right) \quad, \quad \psi^{1}=\frac{\sqrt{2 \pi \alpha^{\prime}}}{g}\left(\begin{array}{c}
\chi_{2} \\
-\chi_{1}
\end{array}\right) .
$$

The spinors $\psi^{0}$ and $\psi^{1}$ satisfy the identities

$$
\begin{gathered}
\bar{\psi}^{b} \rho^{a} \partial_{a} \psi_{b}=0, \\
\bar{\psi}^{a} \psi_{a}=\frac{4 \pi \alpha^{\prime}}{g^{2}} \bar{\chi} \chi
\end{gathered}
$$

where $\chi=\left(\begin{array}{l}\chi_{1} \\ \chi_{2}\end{array}\right)$. Introducing the identity (21) in the action (18) leads to the covariant form of this action

$$
I=-\frac{1}{4 \pi \alpha^{\prime}} \int d^{2} \sigma\left(\partial_{a} X^{M} \partial^{a} X_{M}-i \bar{\psi}^{M} \rho^{a} \partial_{a} \psi_{M}-B^{M} B_{M}\right) .
$$

The metric of the extended manifold is

$$
\eta_{M N}=\operatorname{diag}\left(\eta_{\mu \nu}, \eta_{a b}\right)
$$


where $\eta_{\mu \nu}$ belongs to the $9+1$ actual spacetime and $\eta_{a b}$ for the fictitious coordinates.

The equations of motion, extracted from the action (23), are

$$
\begin{aligned}
& \partial_{a} \partial^{a} X^{M}=0, \\
& \rho^{a} \partial_{a} \psi^{M}=0, \\
& B^{M}=0 .
\end{aligned}
$$

In addition, we should also consider the gauge condition

$$
\partial_{a} X^{a}=0
$$

This condition and the equation of motion of $X^{a}$ can be written as

$$
\begin{gathered}
X^{a}=\varepsilon^{a b} \partial_{b} \phi, \\
\partial_{a} \partial^{a} \phi=c,
\end{gathered}
$$

respectively. The constant $c$ is independent of $\sigma$ and $\tau$.

The fields in the right-hand-sides of the equations (16), (17) and (20) correspond to the gauge theory, while the fields in the left-hand-sides belong to the string theory. The factor

$\frac{\sqrt{2 \pi \alpha^{\prime}}}{g}$ in these relations, for the weak coupling regime in the gauge part, i.e. $g \rightarrow 0$, is equivalent to the zero string tension $\alpha^{\prime} \rightarrow \infty$. This is a result expected from the duality map between gauge theories and strings [4].

\section{Symmetries of the model}

\subsection{Worldsheet supersymmetry}

Using the superfield (8) we obtain the supersymmetry transformations of $A^{a}$ and $\chi$ as in the following

$$
\begin{aligned}
& \delta A^{a}=\bar{\epsilon} \rho^{a} \chi, \\
& \delta \chi=-\frac{i}{4} \rho^{a b} F_{a b} \epsilon-\frac{1}{2} \rho_{a} W^{a} \epsilon, \\
& \delta W^{a}=-i \bar{\epsilon} \rho^{b} \rho^{a} \partial_{b} \chi,
\end{aligned}
$$

where $\rho^{a b}=\frac{1}{2}\left[\rho^{a}, \rho^{b}\right]$. The supersymmetry parameter $\epsilon$ is an anti-commuting infinitesimal constant spinor. For obtaining these transformations the gauge condition (6) has been used. 
In terms of the fields $\left\{X^{a}, \psi^{a}, B^{a}\right\}$ these transformations take the form

$$
\begin{aligned}
& \delta X^{a}=i \varepsilon^{a b} \bar{\epsilon} \psi_{b} \\
& \delta \psi^{a}=-\frac{1}{2}\left(\rho^{a} \varepsilon^{b c} \partial_{b} X_{c}-i \varepsilon^{a b} \rho_{b} \rho_{c} B^{c}\right) \epsilon \\
& \delta B^{a}=\varepsilon^{a b} \bar{\epsilon} \rho^{c} \partial_{c} \psi_{b} .
\end{aligned}
$$

The transformations (30) form a closed algebra. To see this, use the equations of motion to remove $B^{a}$ from these transformations. Now consider two successive transformations with the supersymmetry parameters $\epsilon_{1}$ and $\epsilon_{2}$; then

$$
\left[\delta_{\epsilon_{1}}, \delta_{\epsilon_{2}}\right] X^{a}=\Omega^{a} \varepsilon^{a^{\prime} b^{\prime}} \partial_{a^{\prime}} X_{b^{\prime}},
$$

for the worldsheet bosons $\left\{X^{a}\right\}$, where $\Omega^{a}=i \varepsilon^{a b} \bar{\epsilon}_{1} \rho_{b} \epsilon_{2}$. For the worldsheet fermions $\left\{\psi^{a}\right\}$ we have

$$
\left[\delta_{\epsilon_{1}}, \delta_{\epsilon_{2}}\right] \psi^{a}=i \bar{\epsilon}_{1} \rho^{b} \epsilon_{2} \partial_{b} \psi^{a}
$$

The supercurrent associated to the supersymmetry transformations (30), accompanied by $\delta X^{\mu}=\delta \psi^{\mu}=0$, is

$$
k_{a}=\frac{i}{4} \rho^{a^{\prime}} \rho_{a} \psi_{a^{\prime}} \varepsilon^{b c} \partial_{b} X_{c}
$$

According to the identity $\rho^{a} \rho^{a^{\prime}} \rho_{a}=0$ there is $\rho^{a} k_{a}=0$. That is, some light-cone components of $k_{a}$ vanish. The field equations (25) for $M \in\{0,1\}$, and the gauge condition (26) imply that $k_{a}$ is a conserved current, i.e.,

$$
\partial^{a} k_{a}=0
$$

The transformations (3) and (30) for $\eta=\epsilon$ give the conserved current $J_{a}=j_{a}+k_{a}$.

It is possible to define a two-index current $K_{a b}$ as in the following

$$
K_{a b}=\frac{i}{4} \rho_{a} \psi_{b} \varepsilon^{a^{\prime} b^{\prime}} \partial_{a^{\prime}} X_{b^{\prime}}
$$

In fact, we have $k_{b}=\rho^{a} K_{a b}$. In terms of the fields $A_{a}$ and $\chi$ it becomes

$$
K_{a b}=\frac{\pi \alpha^{\prime}}{4 g^{2}} \rho_{a} \rho^{c} \chi \varepsilon_{b c} \varepsilon^{a^{\prime} b^{\prime}} F_{a^{\prime} b^{\prime}}
$$

This current also satisfies the conservation law

$$
\partial^{a} K_{a b}=0
$$


The action (23) also is invariant under the following supersymmetry transformations

$$
\begin{aligned}
& \delta X^{M}=\bar{\kappa} \psi^{M}, \\
& \delta \psi^{M}=-i \rho^{a} \partial_{a} X^{M} \kappa+B^{M} \kappa, \\
& \delta B^{M}=-i \bar{\kappa} \rho^{a} \partial_{a} \psi^{M},
\end{aligned}
$$

where $\kappa$ is the supersymmetry parameter. The associated conserved current is

$$
\mathcal{J}_{a}=\frac{1}{2} \rho^{b} \rho_{a} \psi^{M} \partial_{b} X_{M}
$$

\subsection{Consistency of the gauge condition with the supersymmetry}

The gauge condition (26) can also be written in the form

$$
\partial_{+} X^{0}+\partial_{+} X^{1}+\partial_{-} X^{0}-\partial_{-} X^{1}=0,
$$

where $\partial_{ \pm}=\frac{1}{2}\left(\partial_{\tau} \pm \partial_{\sigma}\right)$. According to the worldsheet supersymmetry, we should also introduce the fermionic analog of (40), i.e.,

$$
\psi_{+}^{0}+\psi_{+}^{1}+\psi_{-}^{0}-\psi_{-}^{1}=0
$$

where $\psi_{ \pm}^{a}$ are defined by $\psi^{a}=\left(\begin{array}{c}\psi_{-}^{a} \\ \psi_{+}^{a}\end{array}\right)$. According to $(20) \psi_{-}^{a}\left(\psi_{+}^{a}\right)$ has expression in terms of $\chi_{2}\left(\chi_{1}\right)$. Therefore, the equation (41) is an identity. That is, the gauge condition (26) is consistent with supersymmetry.

\subsection{The Poincaré symmetry}

The action (23), with $B^{M}=0$, under the Poincaré transformations

$$
\begin{aligned}
& \delta X^{M}=a^{M}{ }_{N} X^{N}+b^{M}, \\
& \delta \psi^{M}=a^{M}{ }_{N} \psi^{N},
\end{aligned}
$$

is symmetric. The matrix $a_{M N}$ is constant and antisymmetric, and $b^{M}$ is a constant vector. The associated currents to these transformations are

$$
\begin{aligned}
& P_{a}^{M}=\frac{1}{2 \pi \alpha^{\prime}} \partial_{a} X^{M}, \\
& J_{a}^{M N}=\frac{1}{2 \pi \alpha^{\prime}}\left(X^{M} \partial_{a} X^{N}-X^{N} \partial_{a} X^{M}+i \bar{\psi}^{M} \rho_{a} \psi^{N}\right) .
\end{aligned}
$$

These are conserved currents, i.e. $\partial^{a} P_{a}^{M}=\partial^{a} J_{a}^{M N}=0$.

According to the gauge condition (26) the component $J_{a}^{b c}$ takes the form

$$
J_{a}^{b c}=\frac{1}{2 \pi \alpha^{\prime}}\left(-\varepsilon^{b c} \varepsilon_{a a^{\prime}} X^{b^{\prime}} \partial_{b^{\prime}} X^{a^{\prime}}+i \bar{\psi}^{b} \rho_{a} \psi^{c}\right) .
$$




\section{The fictitious coordinates and electric fields}

\subsection{Solutions of the fictitious coordinates}

Now we solve the equation of motion

$$
\left(\partial_{\tau}^{2}-\partial_{\sigma}^{2}\right) X^{a}=0
$$

for the cases that the fictitious coordinates $\left\{X^{a}(\sigma, \tau) \mid a=0,1\right\}$ appear as the coordinates of open and closed strings. The variation of the action (23) leads to the following boundary condition for open string solution

$$
\left(\partial_{\sigma} X^{a}\right)_{\sigma_{0}}=0
$$

where $\sigma_{0}=0, \pi$. Therefore, the solutions of the equation (45) are

$$
X^{a}(\sigma, \tau)=x^{a}+l^{2} p^{a} \tau+i l \sum_{n \neq 0} \frac{1}{n} \alpha_{n}^{a} e^{-i n \tau} \cos (n \sigma),
$$

for the open string, and

$$
X^{a}(\sigma, \tau)=x^{a}+l^{2} p^{a} \tau+2 L^{a} \sigma+\frac{i}{2} l \sum_{n \neq 0} \frac{1}{n}\left(\alpha_{n}^{a} e^{-2 i n(\tau-\sigma)}+\tilde{\alpha}_{n}^{a} e^{-2 i n(\tau+\sigma)}\right),
$$

for the closed string. In these solutions there are $l=\sqrt{2 \alpha^{\prime}}$ and $L^{a}=n^{a} R_{a}$, where $n^{a}$ is winding number of the closed string and $R_{a}$ is radius of compactification of the fictitious coordinate $X^{a}$.

These solutions should also satisfy the condition (26). This condition gives

$$
p^{0}=\alpha_{n}^{0}=\alpha_{n}^{1}=0
$$

for the open string, and

$$
\begin{aligned}
& l^{2} p^{0}+2 L^{1}=0, \\
& \alpha_{n}^{1}=\alpha_{n}^{0} \equiv \alpha_{n}, \\
& \tilde{\alpha}_{n}^{1}=-\tilde{\alpha}_{n}^{0} \equiv-\tilde{\alpha}_{n},
\end{aligned}
$$

for the closed string. Introducing (49) and (50) into the solutions (47) and (48) we obtain

$$
\begin{aligned}
& X^{0}(\sigma, \tau)=x^{0}, \\
& X^{1}(\sigma, \tau)=x^{1}+l^{2} p^{1} \tau,
\end{aligned}
$$


as fictitious coordinates of the open string, and

$$
\begin{aligned}
& X^{0}(\sigma, \tau)=x^{0}+2\left(L^{0} \sigma-L^{1} \tau\right)+\frac{i}{2} l \sum_{n \neq 0} \frac{1}{n}\left(\alpha_{n} e^{-2 i n(\tau-\sigma)}+\tilde{\alpha}_{n} e^{-2 i n(\tau+\sigma)}\right), \\
& X^{1}(\sigma, \tau)=x^{1}+l^{2}\left(p^{1} \tau-p^{0} \sigma\right)+\frac{i}{2} l \sum_{n \neq 0} \frac{1}{n}\left(\alpha_{n} e^{-2 i n(\tau-\sigma)}-\tilde{\alpha}_{n} e^{-2 i n(\tau+\sigma)}\right),
\end{aligned}
$$

for the closed string.

\subsection{Various electric fields}

The worldsheet field strength $F_{a b}=\frac{g}{\sqrt{2 \pi \alpha^{\prime}}}\left(\partial_{a} X_{b}-\partial_{b} X_{a}\right)$ can be written as $F_{a b}=-\varepsilon_{a b} E$, where the worldsheet electric field $E$ is

$$
E=\frac{g l^{2} p^{1}}{\sqrt{2 \pi \alpha^{\prime}}},
$$

from the open string solution (51), and

$$
E=\frac{g\left(l^{2} p^{1}+2 L^{0}\right)}{\sqrt{2 \pi \alpha^{\prime}}}
$$

due to the closed string solution (52). These imply that the zero modes of the fictitious coordinates specify the field strength on the worldsheet.

Now we prove that the compactness of the coordinate $X^{1}$ imposes an electric field along it. There is the following relation between the momentum and winding numbers of a closed string [5],

$$
p^{a}=-\frac{1}{\alpha^{\prime}} f_{a_{c}}^{a} L^{a_{c}}
$$

where the index $a_{c}$ refers to the compact directions and $f_{a b}$ is a background field strength. Since the $X^{1}$-direction is compact this relation gives $p^{0}=-\frac{1}{\alpha^{\prime}} f^{0}{ }_{1} L^{1}$. The other form of this equation is

$$
p^{0}=\frac{1}{\alpha^{\prime}} \mathcal{E} L^{1}
$$

where the electric field along $X^{1}$-direction is defined by $\mathcal{E} \equiv f_{01}$. The equation (56) with the first equation of (50) lead to

$$
\mathcal{E}=-1
$$

Therefore, there is a unit electric field in the $X^{1}$-direction. Note that $\mathcal{E} \neq F_{01}$. 


\section{T-duality and quantization of the fictitious coordi- nates}

\subsection{T-duality}

The zero modes $x^{a}, p^{a}$ and $L^{a}$, in terms of the left and right components are

$$
\begin{aligned}
& x^{a}=x_{L}^{a}+x_{R}^{a}, \\
& p^{a}=p_{L}^{a}+p_{R}^{a}, \\
& L^{a}=\alpha^{\prime}\left(p_{L}^{a}-p_{R}^{a}\right) .
\end{aligned}
$$

Under the $T$-duality $x_{L}^{a}, p_{L}^{a}$ and $\tilde{\alpha}_{n}$ do not change, while $x_{R}^{a}, p_{R}^{a}$ and $\alpha_{n}$ change their signs. Thus, the $T$-duality on the fictitious coordinates (52) gives

$$
\begin{aligned}
& X^{\prime 0}(\sigma, \tau)=x^{\prime 0}+l^{2}\left(p^{0} \sigma-p^{1} \tau\right)+\frac{i}{2} l \sum_{n \neq 0} \frac{1}{n}\left(-\alpha_{n} e^{-2 i n(\tau-\sigma)}+\tilde{\alpha}_{n} e^{-2 i n(\tau+\sigma)}\right), \\
& X^{\prime 1}(\sigma, \tau)=x^{\prime 1}+2\left(L^{1} \tau-L^{0} \sigma\right)-\frac{i}{2} l \sum_{n \neq 0} \frac{1}{n}\left(\alpha_{n} e^{-2 i n(\tau-\sigma)}+\tilde{\alpha}_{n} e^{-2 i n(\tau+\sigma)}\right),
\end{aligned}
$$

where $x^{\prime a}=x_{L}^{a}-x_{R}^{a}$.

Assume the following relations between the center-of-mass coordinates $x^{a}$ and $x^{\prime a}$,

$$
x^{\prime 0}=-x^{1}, x^{1}=-x^{0} .
$$

According to this, compare the dual coordinates (59) with the coordinates (52). We observe that

$$
X^{\prime 0}=-X^{1}, X^{1}=-X^{0} .
$$

That is, up to the sign, the fictitious coordinates are $T$-dual of each other. As expected, under twice dualization these coordinates do not change. The compact form of (61) is

$$
X^{\prime a}=-\varepsilon^{a b} X_{b}
$$

Combine this with the equation (27). In terms of the field $\phi$ this dual coordinate is

$$
X^{\prime a}=-\eta^{a b} \partial_{b} \phi
$$

The equations (28) and (63) give $\partial_{a} X^{\prime a}=-c$. Therefore, the dual coordinates are also constrained by this gauge condition. However, the equations (27) and (63) imply that the scalar field $\phi$ is origin of the four coordinates $\left\{X^{0}, X^{1} ; X^{\prime 0}, X^{\prime 1}\right\}$. 
We define the divergence of the gauge field as $\Phi=-\partial_{a} A^{a}$. From the equations (61) we observe that under the $T$-duality $\Phi$ and $F_{01}=\frac{g}{\sqrt{2 \pi \alpha^{\prime}}}\left(\partial_{\tau} X^{1}+\partial_{\sigma} X^{0}\right)$ are exchanged

$$
\Phi \longleftrightarrow F_{01}
$$

In other words, $T$-dual of the field strength is zero. The $T$-dual version of $F_{a b}$ also can be obtained from (54)

$$
F_{a b}^{\prime}=-\varepsilon_{a b} \frac{g\left(2 L^{1}+l^{2} p^{0}\right)}{\sqrt{2 \pi \alpha^{\prime}}} .
$$

The first equation of (50) gives $F_{a b}^{\prime}=0$. This is consistent with the $T$-duality relation (64).

\subsection{Quantization of the extra worldsheet fields}

Since the condition (41) is an identity the worldsheet fermions $\left\{\psi^{a}\right\}$ are not constrained. Thus, in their quantizations there are not new phenomena. For quantizing the bosonic fields $\left\{X^{a}\right\}$ we need to fix the gauge. The gauge fixed Lagrangian is

$$
\mathcal{L}=-\frac{1}{4 \pi \alpha^{\prime}}\left(\partial_{a} X^{b} \partial^{a} X_{b}+\lambda\left(\partial_{a} X^{a}\right)^{2}\right),
$$

where $\lambda$ is constant. In addition to the equation of motion, extracted from this Lagrangian, we should also consider the equation (26), for example see ref. [6]. The result is the same as the case $\lambda=0$.

Since quantization does not admit the open string solution (51), we consider the closed string solution (52). The canonical momentum conjugate to $X^{a}$ is

$$
\Pi^{a}(\sigma, \tau)=\frac{1}{2 \pi \alpha^{\prime}} \partial_{\tau} X^{a}
$$

The canonical commutation relation at equal $\tau$ is

$$
\left[X^{a}(\sigma, \tau), \Pi^{b}\left(\sigma^{\prime}, \tau\right)\right]=i \delta^{a b} \delta\left(\sigma-\sigma^{\prime}\right)
$$

In fact, consistency with the constraint (26) requires $\delta^{a b}$ instead of $\eta^{a b}$. This quantization leads to the following commutation relations

$$
\begin{aligned}
& {\left[x^{0}, p^{0}\right]=\left[x^{1}, p^{1}\right]=i,} \\
& {\left[\alpha_{m}, \alpha_{n}\right]=\left[\tilde{\alpha}_{m}, \tilde{\alpha}_{n}\right]=m \delta_{m+n, 0},} \\
& {\left[\alpha_{m}, \tilde{\alpha}_{n}\right]=0 .}
\end{aligned}
$$


According to the first equation of (50), the commutator $\left[x^{0}, p^{0}\right]=i$ implies that $L^{1} \neq 0$. In other words, the worldsheet field $X^{1}$ always is compact. The worldsheet field $X^{0}$ can be compact or non-compact.

The following nontrivial commutation relations are consequences of the relations (69),

$$
\begin{aligned}
& {\left[X^{0}(\sigma, \tau), X^{1}\left(\sigma^{\prime}, \tau\right)\right]=2 i \alpha^{\prime} \sum_{n=1}^{\infty} \frac{\sin \left[2 n\left(\sigma-\sigma^{\prime}\right)\right]}{n},} \\
& {\left[\Pi^{0}(\sigma, \tau), \Pi^{1}\left(\sigma^{\prime}, \tau\right)\right]=-\frac{i}{2 \pi \alpha^{\prime}} \delta^{\prime}\left(\sigma-\sigma^{\prime}\right)}
\end{aligned}
$$

where $\delta^{\prime}\left(\sigma-\sigma^{\prime}\right)=\partial_{\sigma} \delta\left(\sigma-\sigma^{\prime}\right)$. These commutators do not show the usual noncommutativity. For the usual noncommutativity we should consider $\sigma=\sigma^{\prime}$, which gives zero for the righthand-sides of (70). However, the fictitious coordinates in different points of closed string have a kind of noncommutativity.

\section{Conclusions}

By appropriate definitions for the superfields and covariant derivative on the worldsheet superspace, we studied the superstring theory in the presence of a worldsheet gauge field. This model contains some connections between the string and gauge fields. For the fictitious $1+1$ directions, the worldsheet supersymmetry and the associated currents were obtained. In addition, the Poincaré symmetry was analyzed.

The fictitious open string coordinates only have zero modes. For the closed string, the winding number around the $X^{1}$-direction is proportional to the momentum along the $X^{0}$ direction. This gives a unit electric field along the $X^{1}$-direction. However, the worldsheet electric fields have expressions in terms of the momentum and winding number of the string.

We observed that the fictitious closed string coordinates $X^{0}$ and $X^{1}$ are $T$-dual of each other. In addition, the field strength and the divergence of the gauge field, under the $T$-duality, transform to each other. Therefore, $T$-dual of the field strength vanishes. A worldsheet scalar $\phi(\sigma, \tau)$ defines the fictitious coordinates $X^{0}$ and $X^{1}$ and their $T$-dual coordinates.

We saw that the quantization of the field $X^{0}$ imposes the compactification of the field $X^{1}$. Finally, in the space $\left\{X^{0}, X^{1}\right\}$ we obtained a noncommutativity between the coordinates of two different points of closed string. 


\section{References}

[1] D.Z. Freedman, P. van Nieuwenhuizen and S. Ferrara, Phys. Rev. D13 (1976) 3214; S. Deser and B. Zumino, Phys. Lett. 62B (1976) 335; Phys. Lett. 65B (1976) 369; J. Wess and B. Zumino, Nucl. Phys. B70 (1974) 39; A. Giveon and M. Rocek, Institute for Advanced Study Preprint No., IASSNS-91/84.

[2] M. Cederwall and P.K. Townsend, JHEP 9709 (1997) 003, hep-th/9709002; P.K. Townsend, Phys. Lett. B409 (1997) 131, hep-th/9705160.

[3] R. Dijkgraaf, E. Verlinde and H. Verlinde, Nucl.Phys. B500 (1997) 43, hep-th/9703030.

[4] J. Maldacena, Adv. Theor. Math. Phys. 2 (1998) 231 [Int. J. Theor. Phys. 38 (1998) 1113], hep-th/9711200; P. Haggi-Mani and B. Sundborg, JHEP 0004 (2000) 031; B. Sundborg, Nucl. Phys. Proc. Suppl. 102 (2001) 113; E. Witten, Talk at the John Schwarz 60-th Birthday Symposium, http://theory.caltech.edu/jhs60/witten/1.html; J. Isberg, U. Lindstrom, B. Sundborg and G. Theodoridis, Nucl. Phys. B411 (1994) 122; I.R. Klebanov and A.M. Polyakov, Phys. Lett. B550 (2002) 213.

[5] H. Arfaei and D. Kamani, Phys. Lett. B452 (1999) 54, hep-th/9909167; Nucl. Phys. B561 (1999) 57, hep-th/9911146.

[6] L. Ryder, "Quantum Field Theory", Cambridge University Press, 1985. 